\title{
INERARED SPECKLE INTERFEROMETRY: A SENSITIVE TECHNIQUE FOR PHYSICAL MEASUREMENTS OF UNSEEN COMPANIONS TO NEARBY STARS
}

\author{
D. W. McCarthy, Jr.* \\ Steward Observatory, The University of Arizona \\ Tucson, Arizona, USA
}

Infrared speckle interferometry combines the full resolving power of large telescopes with high photometric sensitivity over the wavelength range 2.2 to 12 microns. Despite improved atmospheric seeing at these wavelengths, seeing fluctuations limit measurement precision. Astrometric companions have been detected with angular separations $>0.1$ arcsec and magnitude differences $<3.7$ mag. Results illustrate seeing limitations and show how the usual position angle ambiguity can be overcome. These measurements yield masses and absolute magnitudes for calibrating the lower main sequence. In some cases, orbital motion is detected. A method of "shift-and-add" enables detection of substellar ( 0.04 to $0.08 \mathrm{M}_{\odot}$ ) companions. Future improvements involving detector arrays and seeing monitors are discussed.

\section{INTRODUCTION}

The extension of optical speckle interferometry to infrared wavelengths is a recent accomplishment. Sibille et al. (1979) first demonstrated that the complete spatial frequency response of a conventional telescope could be obtained in a single measurement in the presence of atmospheric seeing. Selby et al. (1979) employed a similar technique to measure discrete spatial frequencies. Howell (1980) and McCarthy et al. (1982) made further improvements to eliminate systematic errors and to incorporate low thermal backgrounds, on-line reduction techniques, and methods of alleviating atmospheric seeing effects. Recently Mariotti (1983) and Dyck and Howell (1983) have used infrared speckle interferometry to characterize atmospheric seeing. These investigations demonstrate the limitations imposed by seeing and provide fundamental information needed for future improvements in the technique.

* Visiting Astronomer, Kitt Peak National Observatory, which is operated by the Association of Universities for Research in Astronomy, Inc., under contract with the National Science Foundation. 


\section{GENERAL ASPECTS OF SPECKLE INTERFEROMETRY}

The goal of speckle interferometry is to measure the amplitudes and phases of the spatial frequency components in an object's brightness distribution. A telescope transmits these frequencies up to a cut-off imposed by its finite aperture and therefore acts as a transmission filter with a characteristic modulation transfer function (MTF). Defocus and optical aberrations create transmission losses. However, losses due to atmospheric seeing are generally much larger. The amount of atmospheric attenuation of the high spatial frequencies in the MTE is proportional to $\left(D / r_{0}\right)^{2}$, where $D$ is the telescope diameter and $r_{0}$ (Fried's parameter; Fried 1979) represents the diameter over which the rms wavefront distortion is $\sim$ I radian. At optical wavelengths, $r_{0}$ is typically $\simeq 1 \varnothing \mathrm{cm}$ corresponding to $\sim 1$ arcsec seeing $\left(\lambda r_{0}\right)$. Thus the resulting attenuation factor is $>100 \emptyset$.

Achieving even this attenuated transmission requires special observing methods. Exposure times must be short enough to "freeze" atmospheric turbulence. At optical wavelengths these exposures are 10 millisec. Narrow optical bandwidths are necessary to preserve contrast of the speckles. Individual exposures must be processed into power spectra or autocorrelations and then coadded. Signal-to-noise improves only as the square-root of the number of exposures, although sometimes even more slowly since the quantity ro exhibits slow variations which are difficult to calibrate. Sibille et al. (1979) have determined that signal-to-noise is proportional to $r_{0}^{4}$.

The quantity $r_{0}$ has a wavelength dependence $\lambda^{1.2}$. Therefore, at infrared wavelengths, the attenuation factor in the above example is only $\sim 10 \mathrm{x}$ and allowable exposure times and filter bandwidths are $10 \mathrm{x}$ larger. For values of $r_{0} \simeq D$, the point spread function approximates the diffraction limit.

\section{THE INFRARED SPECKLE TECHNIQUE}

Observing Procedures and Data Reduction

At the Steward Observatory 2.3-m telescope and the Kitt Peak National Observatory 3.8-m telescope, speckle exposures are obtained by scanning the Cassegrain infrared secondary mirror ( $f / 28-45)$ to generate linear scans of stellar images in the focal plane. The scan rate ( 60 arcsec/sec) is sufficient to "freeze" the effects of atmospheric turbulence on the image as it is scanned across a narrow slit in front of a single detector. The slit acts as a spatial frequency filter and has an angular width $\geq \lambda / D$. Successive scans are converted on-line into power spectra which are coadded to improve signal-to-noise. Observations proceed by alternating blocks of $6 \emptyset 0$ scans ( $1 \emptyset \emptyset \mathrm{sec}$ ) on an object of interest and on a nearby calibration "point" source which provides a measure of the instrumentalatmospheric transfer function. The average of alternate power spectra 
of the calibration source is used to normalize the spectrum of the program object. The square-root of this ratio is the visibility function of the program object. Successive visibility measurements are coadded to reduce the mean errors. This method of observation, although tedious and demanding in its need for frequent, rapid offsets of the telescope, is effective in reducing the effects of slow changes in $r_{0}$ which occur over periods of a few minutes.

Different scanning angles and slit orientations are used to sample the two-dimensional visibility function. Different wavelengths, bandwidths, slit widths, and scan speeds are selectable to optimize signal-to-noise for different objects and for different atmospheric conditions.

Figure 1 displays visibility measurements of the astrometric binary Ross 614 obtained with the 2.3-m telescope. These observations yield the angular separation ( $0.57 \mathrm{arcsec}$ ) and brightness ratio (1.7 mag). The position angle cannot normally be determined because of the power spectrum analysis. This obstacle can be overcome by multiplying

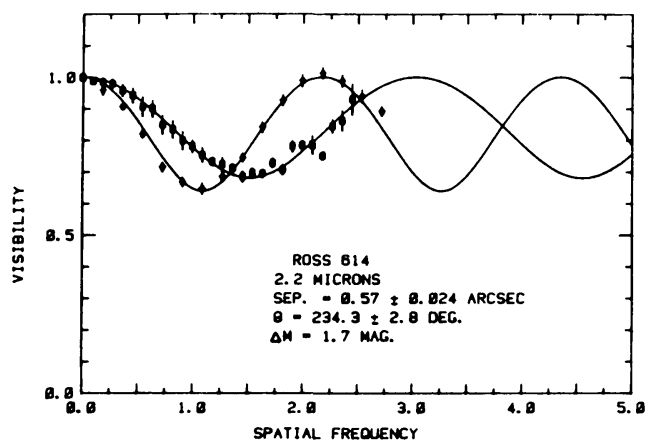

Fig. 1. Visibility measurements of Ross 614 at 2.2 microns in two orthogonal directions providing angular separation and brightnesses.

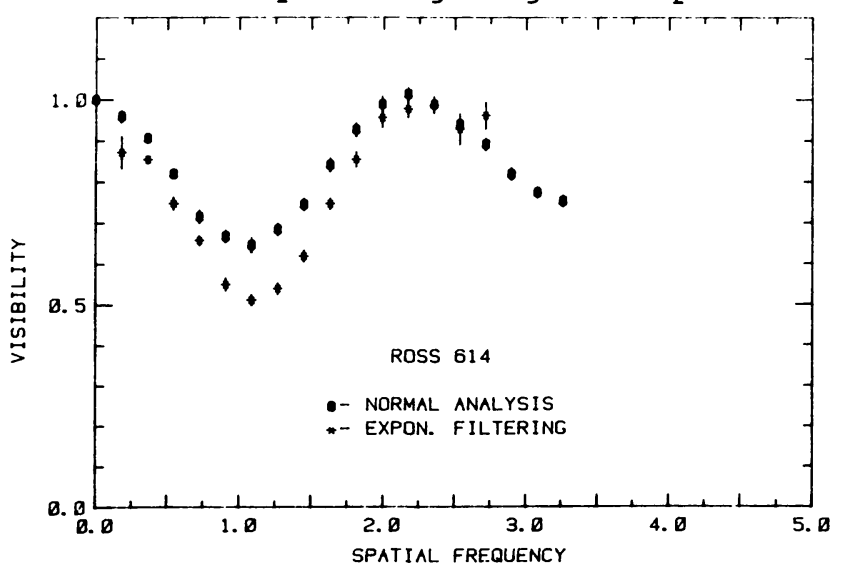

Fig. 2. Visibility measurements of Ross 614 at 2.2 microns with and without exponential filtering for determining the position angle. 
the original scans by an exponential function and then continuing the normal analysis (Walker 1982). The resulting visibility function yields a different brightness ratio (Figure 2) which reveals the orientation of the faint component relative to the known exponential.

\section{Atmospheric Limitations}

Figures 3 and 4 illustrate some harmful effects of atmospheric seeing. Figure 3 displays successive scans of the visual binary 70 Oph (projected separation 2.2 arcsec) at $2.2 \mathrm{microns}$ obtained at the 3.8-m telescope. Irregularities in the stellar profiles correspond to speckles in the images. Atmospheric seeing changes these profiles between scans but, within each scan, the two profiles should be identical apart from a scale factor since the two stars lie within the isoplanatic patch. However, inspection of the individual scans (e.g., scans 3,7$)$ reveals changes in the relative brightness and separation of the two stellar profiles. Apparently the scan rate (42 arcsec/sec) is too slow to freeze turbulence effects over a $\sim 3$ arcsec region (star separation plus seeing width). This effect

SPECKLE SCANS OF 7D OPH

\section{2 MICRONS 4-METER TELESCOPE \\ SCAN LENGTH $=7$ ARCSEC \\ SCAN DURATION = 0. 167 SEC}

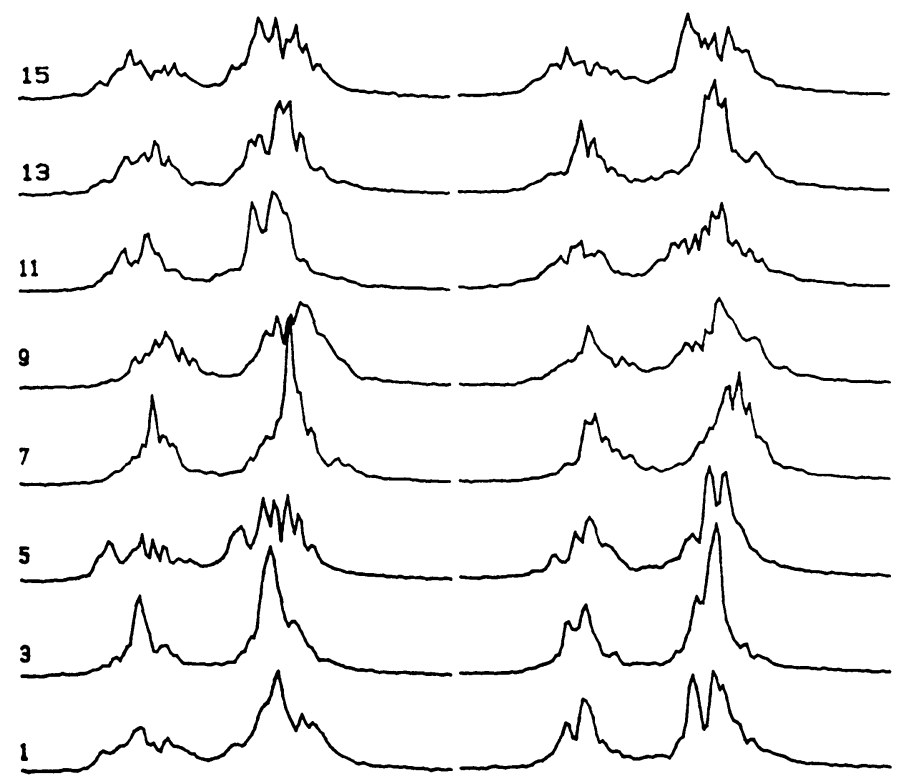

Fig. 3. Speckle scans of the visual binary $7 \emptyset$ oph at $2.2 \mathrm{microns}$ with the Mayall 3.8-m telescope. Scan rate is 7 arcsec in 0.167 sec. Successive scans are separated by $0.33 \mathrm{sec}$. Scan-to-scan variations in the separation and relative brightness of the two stars indicate that this scan rate is too slow to freeze atmospheric turbulence. 
modulates the sinusoidal visibility function expected from a double star and simulates the presence of additional sources. Accurate speckle measurements of such wide binaries require either faster scan rates or scan angles perpendicular to the separation vector where the image size is much smaller.

Figure 4 illustrates visibility measurements of the astrometric binary Alpha Oph at 2.2 microns during a night of subarcsec visual seeing. The periodicity in visibility is obvious and demonstrates that large magnitude differences (3.7 mag) can be measured. However, the visibilities rise above unity and systematic errors appear to dominate over random errors. These characteristics are probably caused by slow variations in atmospheric seeing occurring during the interval between measurements of the program object and the calibration source. This effect is currently the dominant source of error in the infrared speckle technique and limits accuracy in absolute visibility measurements to $\sim 5$ percent. Improvements in this area will result when scans with similar $r_{0}$ can be selected on-line and processed independently or with different weighting factors.

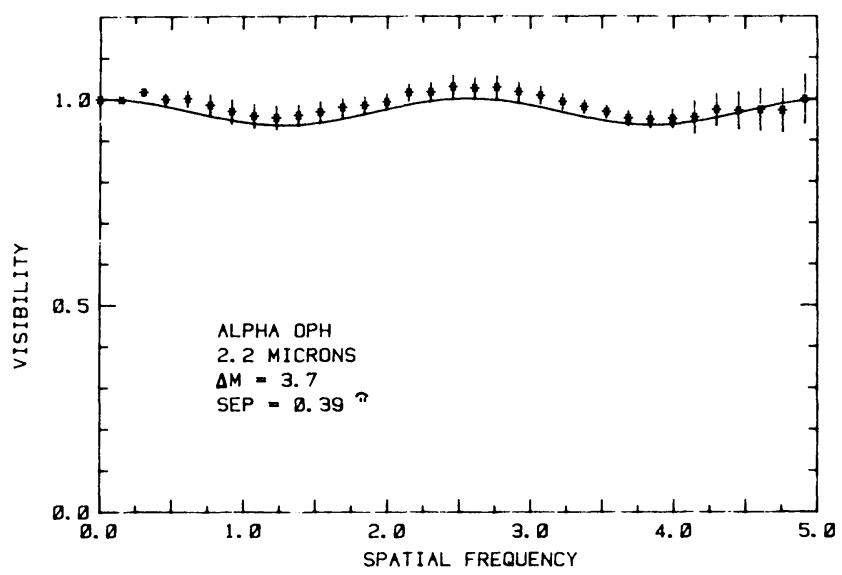

Fig. 4. Visibility measurements of the astrometric binary Alpha Oph at 2.2 microns with the $2.3-\mathrm{m}$ telescope. These results demonstrate that large magnitude differences can be measured and also illustrate the systematic effects of slow changes in atmospheric seeing.

\section{ASTROMETRIC APPLICATIONS}

Infrared speckle interferometry is a powerful tool for detection of low mass stellar and substellar companions to nearby stars. Lippincott (1978) has summarized studies of the $\sim 25$ astrometric companions investigated over the last $4 \emptyset-5 \emptyset$ years. The expected angular separations range from $\emptyset .1$ to $\sim 1$ arcsec and most objects are expected to be red dwarfs with emission peaks in the near-infrared. 
Infrared speckle measurements of these objects have detected companions in 14 objects (McCarthy et al. 1982; McCarthy 1933). Previous efforts at optical wavelengths resolved only two objects because of the large magnitude differences between sources.

The infrared measurements yield angular separations and apparent magnitudes. When combined with existing astrometric measurements (photocentric orbital elements and absolute parallaxes), this information provides individual masses and absolute magnitudes of the component objects. These results provide new empirical knowledge of the lower main sequence and the local luminosity function. In specific cases, other astrophysical problems can be studied such as the evolution of the galactic helium abundance (McCarthy 1984).

Considerable care must be exercised in combining the infrared and astrometric measurements. Since the measured angular separation, photocentric separation, orbital period, and parallax all contribute to the mass determination through Kepler's Third Law, errors in these quantities are magnified in the final result. In some cases (Alpha Oph, Wolf 922, etc.) it is apparent that errors in the orbital elements lead to inaccurate photocentric separations. These errors may result from measurement inaccuracies, from the necessity to predict orbital positions by extrapolation of the astrometric orbit (especially important for short period binaries), and from the presence of additional companions not detected astrometrically. The infrared absolute magnitudes provide checks on the derived masses; the measured position angles provide checks on the predicted positions.

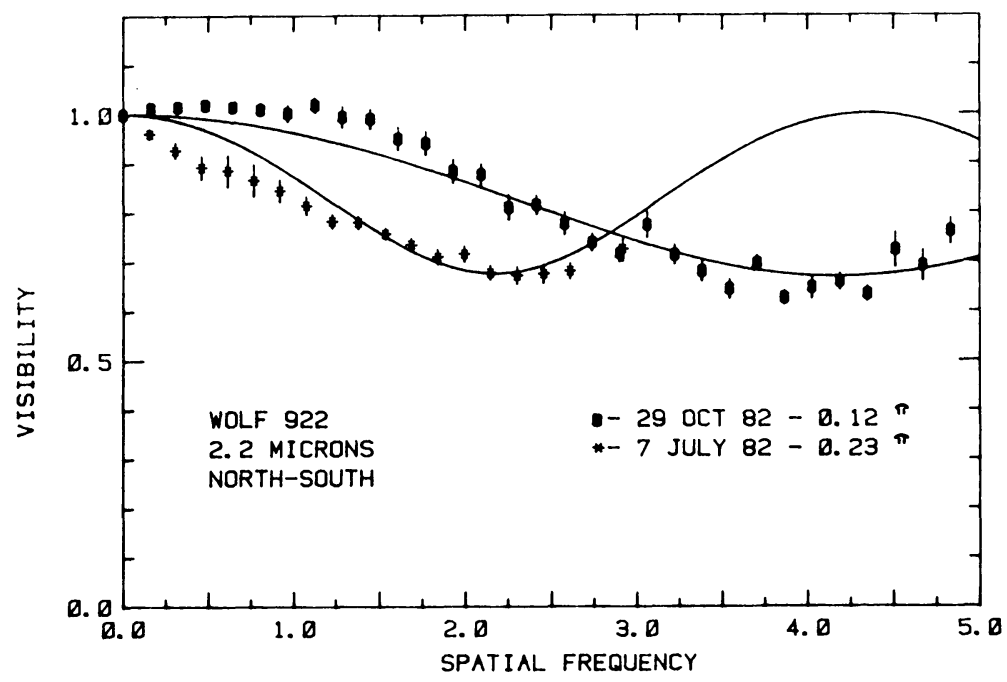

Fig. 5. Visibility measurements of the astrometric binary Wolf 922 at 2.2 microns with the 2.3-m telescope. Measurements over a four month interval reveal orbital motion in this short period (1.9 year) object. 
For short period binaries it is already possible to detect orbital motion from the infrared speckle measurements. Figure 5 illustrates one-dimensional separation measurements of the astrometric binary Wolf 922 (period 1.9 years) showing a factor of two change in separation over a four month period. Such observations will ultimately lead to independent sets of orbital elements for comparison with conventional astrometry.

Table I summarizes the astrometric companions detected by infrared speckle interferometry. Masses are derived either as discussed above or, when the predicted position appears inaccurate, they are calculated from the measured infrared absolute magnitudes using existing mass-luminosity relationships (Staller and de Jong 1981; Veeder 1975).

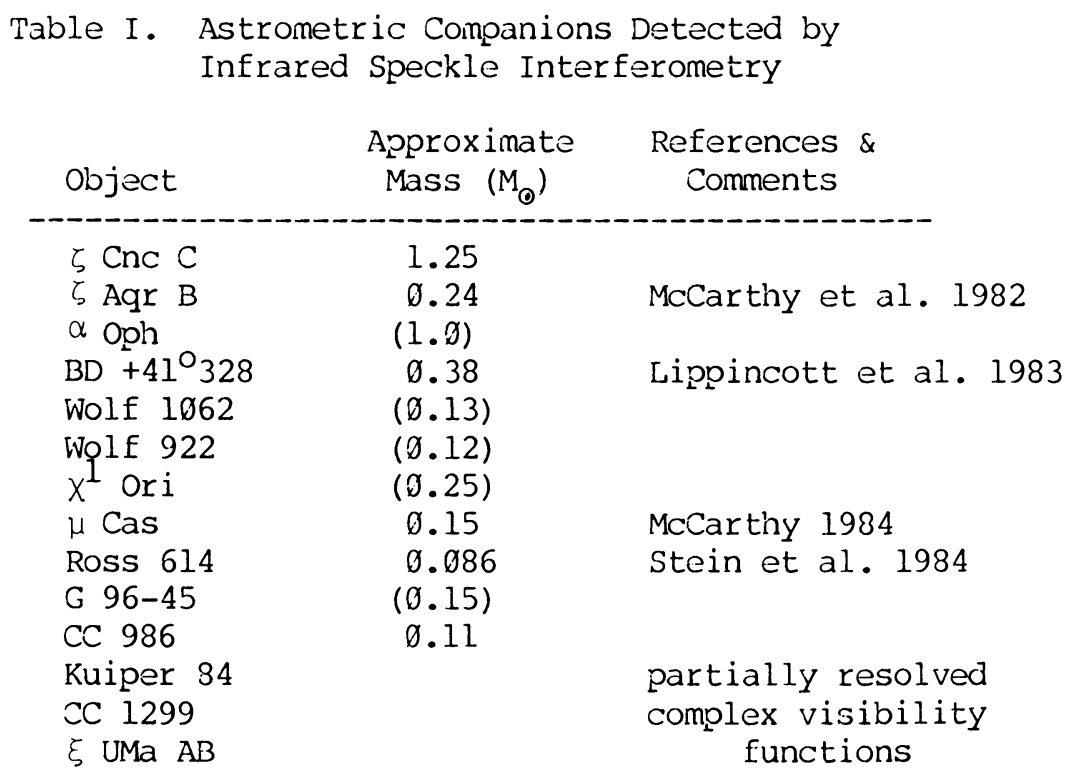

\section{Removal of Ambiguities in Conventional Astrometry}

Infrared speckle measurements help eliminate possible ambiguities in existing astrometric measurements. For example, bright companions may be undetected astrometrically if the photocenter and center-ofmass are coincident. Also companions with periods shorter than the usual exposure frequency may have been missed in previous studies.

Therefore it is significant that the infrared measurements do not reveal companions in the objects suspected of having planetary companions. Since these companions should presently be undetectable in the near-infrared, the infrared speckle measurements actually strenghten evidence in favor of planetary companions. However, the 
photocentric perturbations in these systems are quite small and it is also possible that the primary stars are single. Table II lists the objects which are unresolved by the infrared speckle work but which have suspected planetary companions.

Table II. Astrometric Companions not Detected by Infrared Speckle Interferometry

\begin{tabular}{|c|c|}
\hline & Suspected \\
\hline Object & Mass $\left(M_{0}\right)$ \\
\hline Barnard's Star & $\emptyset . \emptyset \emptyset \emptyset 8 / \varnothing . \varnothing \emptyset \emptyset 4$ \\
\hline CC 1228 & $>0.02$ \\
\hline Stein 2051A & $\overline{>0 . \emptyset 2}$ \\
\hline $\mathrm{BD}+43^{\circ} 4305$ & $\overline{>} . .009$ \\
\hline $\mathrm{BD}+68^{\circ} 946$ & $\geq \varnothing .0 \emptyset 6$ \\
\hline G 24-16 & $\overline{>0.07}$ \\
\hline
\end{tabular}

\section{SHIFT-AND-ADD: SEARCHING FOR SUBSTELI_AR COMPANIONS}

Objects with masses below the theoretical lower mass limit for hydrogen burning $\left(\leq 0 . \emptyset 8 M_{\odot}\right)$ should be detectable by infrared speckle interferometry. Stevenson (1978) has calculated that objects of solar system age with masses from $\emptyset . \emptyset 4$ to $\emptyset . \emptyset 8 \mathrm{M}_{\odot}(4 \emptyset-8 \emptyset$ Jupiter masses) will have temperatures between $\sim 8 \emptyset \emptyset$ and $1200 \mathrm{~K}$. Their thermal radiation should peak at wavelengths of 2.2 to 3.4 microns. Since their predicted absolute magnitudes at $2.2 \mathrm{microns}$ are 10-12 mag, infrared speckle interferometry could detect such objects as companions to stars within $\sim 1 \emptyset$ parsecs of the Sun. However, it is essential to select faint, low mass primaries in order to minimize the magnitude differences in these systems. Differences larger than $\sim 3.5$ mag will probably be masked by atmospheric effects.

One method for achieving the desired sensitivity and for reducing the effects of atmospheric seeing is the method of shift-and-add (Bates 1982). The idea is first to coadd successive scans by aligning their intensity peaks. Frequent observations of a program object and a calibration object are obtained as discussed previously. Coaddition of scans proceeds until the noise level is reduced considerablyperhaps to the level of digitization noise. The resultant image profiles are then deconvolved to reveal the presence of faint sources around the program object. The CLEAN algorithm (Hogbom 1974) provides a deconvolution method known to work well on systems of discrete sources. Residuals from the deconvolution limit the achieveable sensitivity. This shift-and-add technique should work efficiently in the infrared where the number of speckles in an image is relatively small and the peak intensity will be well defined.

Figures 6 and 7 show initial efforts to apply this procedure to nearby stars. Figure 6 illustrates results on the binary Luyten 726-8, 

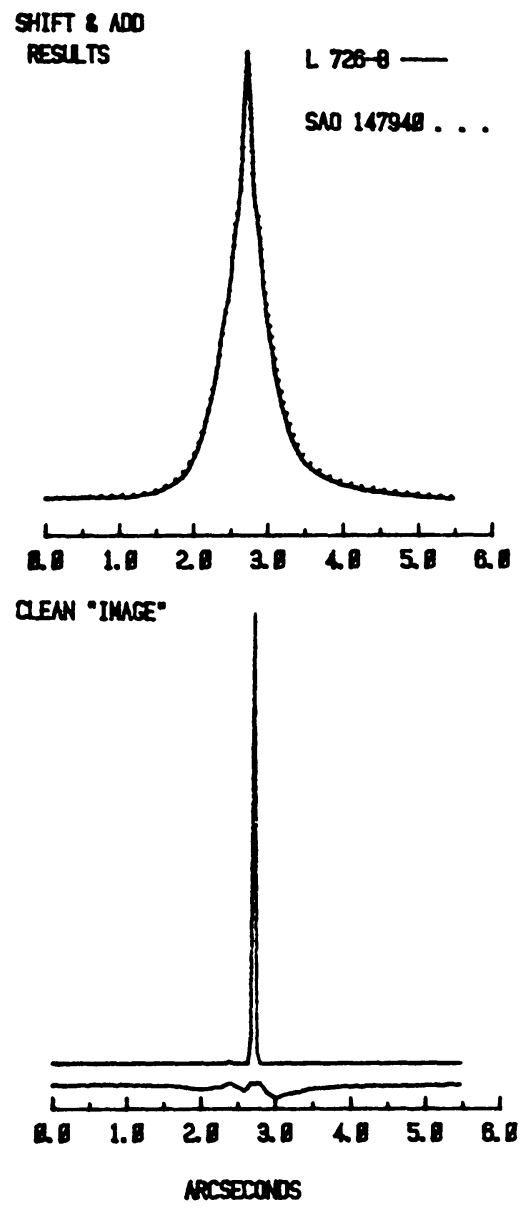

Fig. 6. The shift-and-add method applied to L 725-8. The object is scanned perpendicular to the separation of the visual binary. No substellar companions are detected. Residuals from the CLEAN process are shown beneath the final image.
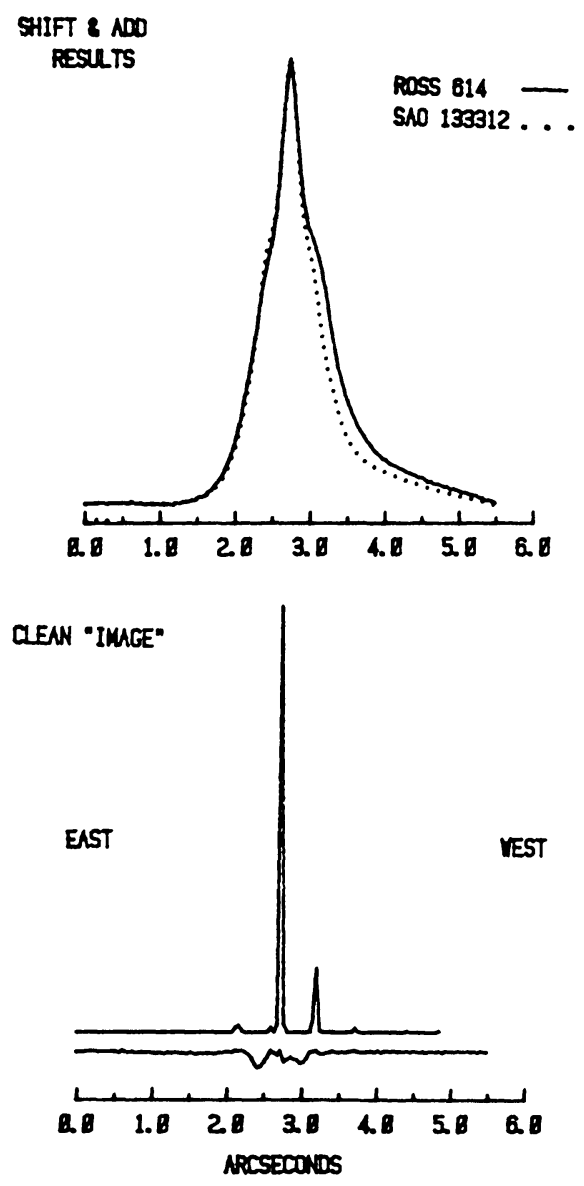

Fig. 7. The shift-and-add method aplied to Ross 614. The astrometric companion is resolved but no objects with masses $\emptyset . \emptyset 4-\emptyset . \emptyset 8$ $M_{\odot}$ are detected. Residuals from the CLEAN process are shown below the final image.

a pair of dwarf stars with masses $0.1 \mathrm{M}_{\odot}$. A $7 \times 7$ arcsec region was scanned centered on the binary but in a direction perpendicular to its separation vector. The CLEANed "image" shows a single peak representing both stars. No companions are observed with absolute magnitudes brighter than 12 mag thereby excluding the existence of substellar companions with masses $0.04-0.08 \mathrm{M}_{\odot}$ and $\mathrm{with}$ ages of $<5$ billion years. Figure 7 illustrates results on the astrometric binary Ross 614. The binary is easily resolved and similar limits are placed on the existence of substellar companions. 


\section{FUTURE IMPROVEMENTS}

Major improvements in the technique of infrared speckla interferometry involve the application of two-dimensional detector arrays and the application of an on-line seeing monitor during observations. Detector arrays will eliminate the present need for image scanning and provide constant illumination of detectors. They will also eliminate the focal plane slit and the associated diffraction losses, aberrations, and averaging of speckles. In addition, all position angles will be sampled simultaneously. All these features will lead to sizeable gains in observing efficiency. An on-line seeing monitor will allow selection of scans obtained under identical seeing conditions. Individual power spectra can then be coadded with weighting factors or coadded in separate bins for independent processing. This feature should reduce the degree of sensitivity to seeing variations.

\section{ACKNOWLEDGEMENTS}

This program is supported by the National Science Foundation under grant AST 8218782. The author thanks Drs. F. J. Low and S. G. Kleinmann for help in accumulating these observations and for stimulating discussions. Encouragement from P. A. Strittmatter, E. K. Hege, and R. Probst is also acknowledged. Drs. G. Rieke, M. Lebofsky, and R. Joyce provided detector systems for use in this project.

\section{REFERENCES}

Bates, R. H. T., and Fright, W. R.: 1982, Monthly Notices Roy. Astron. Soc., 198, 1017.

Dyck, H. M., and Howell, R. R.: 1983, Publ. Astron. Soc. Pacific, 95, 786.

Fried, D. L.: 1979, in J. Davis and W. J. Tango (ed.) High Angular Resolution Stellar Interferometry: Proc. of I.A.U. Collog. No. 50, Sydney, p.4-1.

Hogbom, J. A.: 1974, Astron. \& Astrophys. Supl., 15, 417.

Howell, R. R.: 1980, Ph.D. Dissertation, The University of Arizona.

Lippincott, S. L.: 1978, Space Sci. Rev., 22, 153.

Lippincott, S. L., Braun, D., and McCarthy, D. W.: 1983, Publ. Astron. Soc. Pacific, 95, 275.

Mariotti, J. M.: 1983, Optica Acta, 36, 831. 
McCarthy, D. W., Low, F. J., Kleinmann, S. G., and Arganbright, D. V.: 1982, Astrophys. J. (Letters), 257, L75.

McCarthy, D. W.: 1983, in The Nearby Stars and the Stellar Luminosity Function: Proc. of I.A.U. Colloq. No. 76, in press.

McCarthy, D. W.: 1984, Astron. J., in press.

Selby, M., J., Wade, R., and Magro, C.: 1979, Monthly Notices Roy. Astron. Soc., 187, 553.

Sibille, F., Chelli, A., and Lena, P.: 1979, Astron. \& Astrophys.,79 315.

Staller, R. F. A., and de Jong, T.: 1981, Astron. \& Astrophys., 98, 140 .

Stein, J. W., Kamper, K. W., and McCarthy, D. W.: 1984, in preparation.

Stevenson, D. J.: 1978, Proc. Astron. Soc. Australia, 3, 227.

Veeder, G. J.: 1975, Astron. J., 79, 1056.

Walker, J. G.: 1982, Appl. Opt., 21, 3132.

Discussion:

EICHHORN: What is the largest magnitude difference you can handle?

MCCARTHY: I agree with McAlister in saying it is about $4 \mathrm{~m}$. We may be able to detect periodicities when the magnitude difference is $5^{\mathrm{m}}$, but for absolute measurements this will be impossible. Barnard's star's companion, for instance, is not within our reach.

GLIESE:

MCCARTHY:

HEINTZ:
Are spectroscopic doubles included in the Aitken catalogue? Optical ones, but not infrared ones.

Among several systems I have currently under astrometric study a recent result on $\xi$ Aqr may be mentioned. The orbit scale of the unresolved companion from relative observations is $75 \pm 3$ mas; the astrometric positions give about $90 \pm 20$ mas for star $A,-20 \pm 20$ for star B, indicating that the 26-year period is in star A.

MeCARTHY:

I am aware of optical speckle measurements indicating a companion to star A; the infrared observations clearly indicate something around star B from the Arizona and the (unpublished) Hawaii measurements. 